\section{Bois et Forêts des Tropiques contribue à la médiation scientifique pour accompagner les changements indispensables de la société}

Les connaissances et les savoirs sont essentiels pour faire évoluer les sociétés. Ils constituent un élément-clé pour mieux cerner le monde qui nous entoure et mieux comprendre les changements et les défis auxquels nous devons faire face. Le métier de chercheur consiste à exploiter ce gisement intarissable représenté par l'inconnu. Les chercheurs apportent au grand public cette ressource en la modelant de façon à la rendre accessible aux diverses strates de la société. Malgré la multitude de formes imaginées pour interagir, il s'avère que ces dialogues peinent encore à tisser les liens solidaires indispensables entre les différentes parties prenantes pour transformer ensemble le monde. Les citoyens se sentent bien souvent exclus et les scientifiques ont le sentiment de ne pas toujours être bien entendus.

La communication ou médiation scientifique, notamment celle liée aux sciences du bois, sciences forestières et sciences de l'environnement, doit aujourd'hui amener à considérer tout public en tant qu'acteur essentiel, en l'invitant à participer aux réflexions scientifiques. Cette ouverture vers le grand public aidera à mieux le préparer aux évolutions de son environnement scientifique tout en développant une pensée critique et rationnelle. Cela amènera tous les citoyens à participer de manière responsable aux grands enjeux sociétaux et environnementaux. C'est donc en introduisant la science dans la société, et vice versa, que les évolutions scientifiques et sociétales seront mieux acceptées. Le travail des chercheurs ne se résume pas à la publication de leurs expériences et de leurs résultats dans des articles ou tout autre support destiné et réservé uniquement aux univers académiques et scientifiques. Ce travail de partage de connaissances et savoirs attire le chercheur bien au-delà de cette sphère d'évaluation et de publication nécessaire à la profession. Les chercheurs trouvent des occasions et des solutions pour familiariser le grand public à la démarche scientifique.

C'est dans cette perspective que Bois et Forêts des Tropiques s'associe à cette démarche de médiation scientifique et de diffusion des savoirs au sein de la société et vers le grand public. Cette action se traduit par la mise en avant de quelques exemples de l'implication de chercheurs des sciences du bois et des sciences forestières. Il est essentiel que le grand public prenne connaissance du fonctionnement des forêts, de leur utilité pour l'environnement, ainsi que des multiples services qu'elles rendent à l'humanité. Pour mieux comprendre tous ces phénomènes observés et les méthodes scientifiques employées, le citoyen a besoin de pratiquer l'expérimentation par une mise en situation. Il s'impliquera ainsi plus facilement dans des réflexions collectives relatives aux grands enjeux planétaires et locaux, pour lesquels chacun d'entre nous joue un rôle, qu'il s'agisse de la conservation de la biodiversité, de la protection et de la valorisation des forêts, ou de l'atténuation du changement climatique.

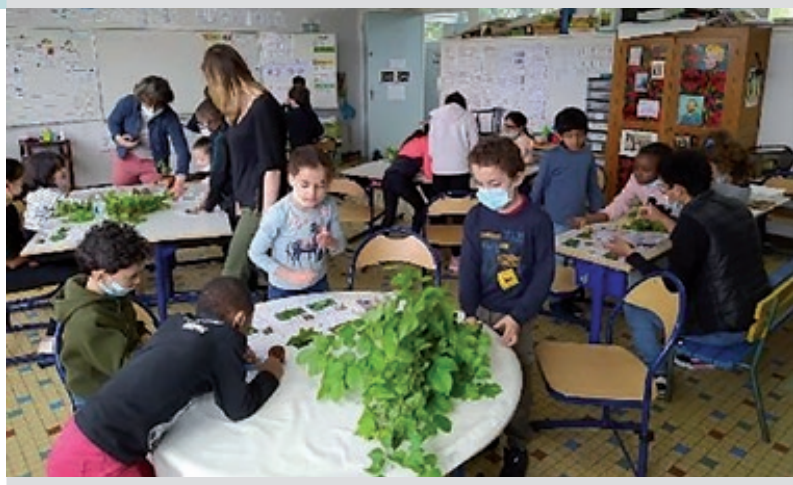

Identification et reconnaissance des principales essences d'arbres présentes dans les forêts françaises. Atelier mené en classe et encadré par une guide "nature et patrimoine » et un chercheur en sciences du bois, en partenariat avec le dispositif «Savanturiers - École de la Recherche». Photo K. Candelier.

Cet engagement sociétal se manifeste par exemple lorsqu'un chercheur intervient auprès d'un jeune public, en accompagnant des enfants à découvrir la démarche scientifique et les postures adoptées dans son travail. C'est ce que nous rapporte l'expérience menée par Candelier et al., en partenariat avec le dispositif "Savanturiers - École de la Recherche ", publié dans ce numéro de Bois et Forêts des Tropiques. À cette occasion, les auteurs nous décrivent la fraîcheur du candide face à l'inconnu et les nouveaux savoirs qu'il tente de faire émerger et d'intégrer avec méthode et rigueur, encadrés par un chercheur. Ce qu'ils nous disent, c'est que cet échange entre la sphère scientifique et la société doit s'adosser aux principes d'une éducation aux sciences et à la démarche scientifique, à un accès équitable à la connaissance, mais aussi, et surtout, à l'incontournable entrelacement collaboratif des différents

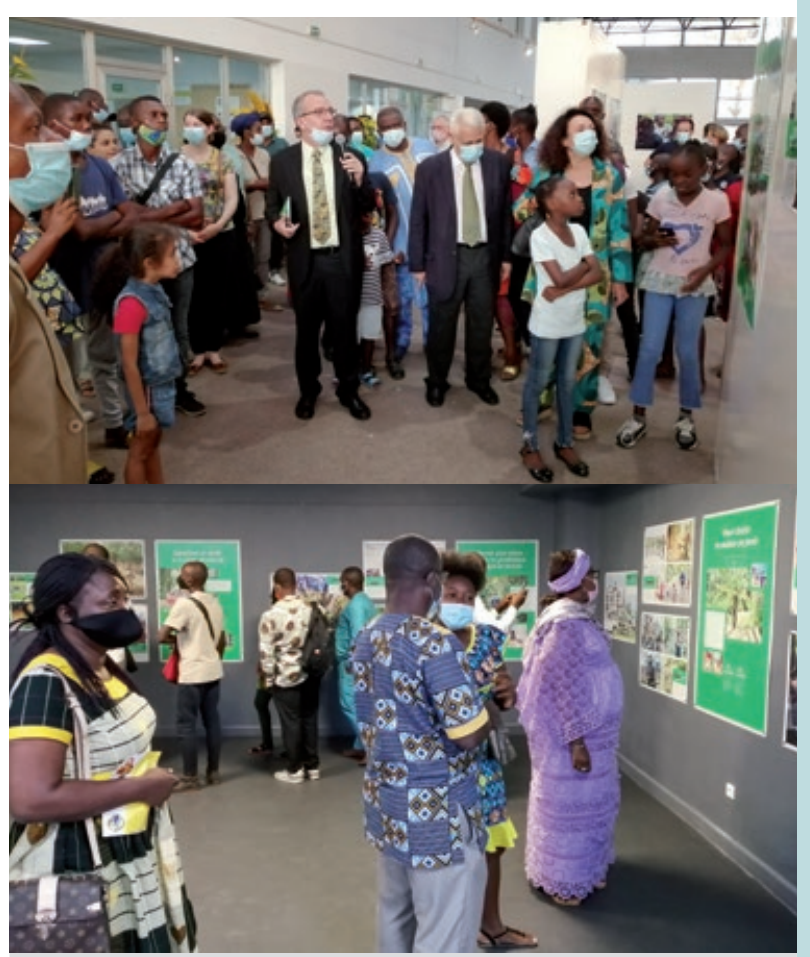

Exposition photographique itinérante « L'avenir des forêts est entre vos mains! » (Rossi V., Lescuyer G., 2021).

Photos Collectif de recherche Forêts et Sociétés, Cirad. 
Bois et Forêts des Tropiques - ISSN: L-0006-579X

acteurs en quête de savoirs. Dans ce cas, le chercheur et l'enseignant inscrivent l'élève dans une démarche de pédagogie collaborative tout en le rendant acteur de son auto-apprentissage. Elle déclenche chez l'apprenant les mécanismes pour observer, expérimenter, exposer son jugement et le discuter à travers des jeux, des mises en scène, des ateliers, et via des expériences sensorielles, sociologiques, écologiques.

Les possibilités de transmission de savoirs vers la société sont sans limites et parfois même imperceptibles. Elles peuvent apparaître dans des festivals, des expositions, des ateliers pédagogiques, des randonnées nature, dans la presse quotidienne, dans des films, des livres, les bandes dessinées, etc. Les chercheurs sont libres de faire jouer leur imagination et d'utiliser à loisir les médias afin de mieux se connecter avec le citoyen. Cette médiation peut prendre la forme d'une exposition itinérante sur la manière de préserver et de valoriser les forêts d'Afrique centrale, telle qu'organisée par Rossi et Lescuyer (2021), ou un livre adressé au grand public sur les pistes possibles de préservation et de valorisation des forêts tropicales, tel Vivre avec les forêts tropicales rédigé et illustré par le collectif de recherche Forêts et Sociétés (Sist et al., 2021), ou encore un jeu de rôle comme Foster forest (Fouqueray, 2019 et 2020) pour adapter les pratiques professionnelles de la foresterie afin de faire face aux changements climatiques, produit émergeant du parcours d'un doctorant encadré par une équipe de chercheurs. L'énumération serait longue tant elle est foisonnante. Les scientifiques encouragent les différentes strates de la société à s'approprier les savoirs et les connaissances qui les amèneront à prendre des décisions individuelles et collectives éclairées. Par le canal médiatique de Bois et Forêts des Tropiques, ils promeuvent les produits de la recherche autrement que les articles qui y sont publiés traditionnellement. C'est pourquoi paraissent dans les pages de cette revue scientifique et technique des résumés de thèse et des descriptifs techniques. Dans l'avenir, les chercheurs pourront occasionnellement s'y exprimer sous d'autres formes encore, comme l'ont proposé Candelier et al. (2021). Ces efforts de médiation aident à réagir face aux petits et grands défis qui tapissent les paysages de l'environnement dans lesquels nous évoluons tous, tels que les grands changements sociétaux liés à la démographie, le partage des ressources et savoirs, les changements des climats, pour ne citer que ceux-là. L'exposé de ces quelques exemples d'entrelacement sociétal témoignent donc de l'implication quotidienne de la recherche dans la société, et vice versa.

Kévin CANDELIER, Jean-François TrÉBuchoN Membres de l'équipe éditoriale de la revue Bois et Forêts des Tropiques

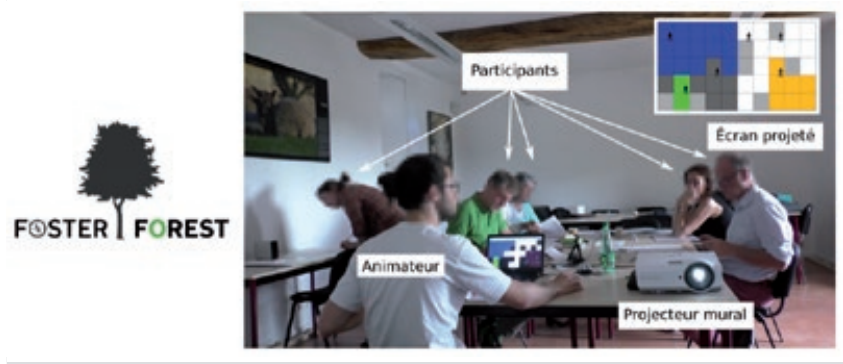

Foster Forest, jeu sérieux de simulation participative pour l'adaptation aux changements climatiques des forestiers. Cet atelier ludique permet à des forestiers publics, des propriétaires privés, des experts forestiers et des gestionnaires d'espaces naturels de tester ou d'imaginer des modes d'adaptation aux dérèglements climatiques en forêt (Fouqueray, 2020). Photo F. Fouqueray.

\section{Références}

Candelier K., Mouelle P., Ocana A., Batteux M., Manzanares E., Clair P., Ansour A., 2021. Accompagner la découverte scientifique des arbres par de jeunes élèves (Creil, France). Bois et Forêts des Tropiques, 349 : 85-94. Doi : https://doi. org/10.19182/bft2021.349.a36792

Fouqueray T., 2019. Adaptations aux incertitudes climatiques de long terme : trajectoires socio-écologiques de la gestion forestière française. Thèse de doctorat, Université de Paris-Saclay, $295 \mathrm{p}$.

Fouqueray T., 2020. Foster forest. Jeu de rôle sérieux de simulation prospective. http://www.fosterforest.fr/

Sist P., Doumenge C., Gond V., Tassin J., Trébuchon J.-F. (eds), et al., 2021. Vivre avec les forêts tropicales. Éditions MUSEO, $216 \mathrm{p}$.

Rossi V., Lescuyer G., 2021. L'avenir des forêts est entre vos mains! Cirad, exposition photographique itinérante.

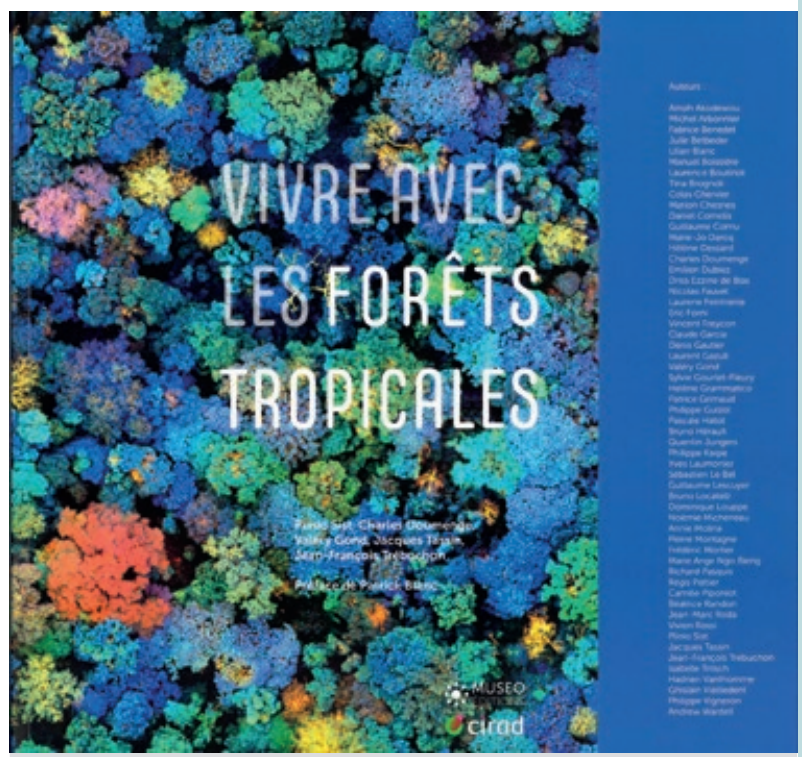

Couverture du livre Vivre avec les forêts tropicales, conçu et rédigé par un collectif de chercheurs. Le livre est à rebours des idées reçues, sans jargon ni condescendance pour mieux s'adresser au grand public francophone. Il rend compte des imbrications respectives entre les humains et les forêts tropicales (Sist et al., 2021). 